\title{
Study of Candidate Insulating Materials for Use in Shock Wave Switches
}

\author{
S.V. KolosenoK ${ }^{a, *}$ And L.L. Altgilbers ${ }^{b}$ \\ ${ }^{a}$ Sankt-Petersburg State University \\ 1, Ulyanovskaya str., Petrodvorets, Sankt-Petersburg, 198504, Russia \\ ${ }^{b}$ Advanced Technology Directorate, U.S. Army Space and Missile Defense Command
}

Huntsville, AL, USA

\begin{abstract}
One challenge in high power switching is to have a compact switch, which can hold off high voltages and close rapidly at the proper time. Most high power switches are large or complicated, such as triggered spark gaps. Typical opening switches are also not compact and often have too long switching time. It has been shown previously that certain insulating materials undergo a drastic change in conductivity under shock loading. Using such a material could greatly reduce the size of a switch. We will report on our continuing studies of different candidate materials for shock wave switches.
\end{abstract}

PACS numbers: 77.22.Jp, 77.80.Fm, 77.84.Lf, 81.05.Qk, 81.05.Rm, 81.65.-b

\section{Introduction}

In our previous paper we published the results of pulsed DC breakdown testing of commercially available materials, suitable for the shock wave switching [1]. Now we continue our studies with investigation of laboratory prepared metal-dielectric composite materials, based on aluminum powder.

\section{The description of test setup}

Here, we present the results of a brief study of the electrical breakdown strength of a few composite materials, prepared from aluminum powder with addition of insulation. The aluminum powder we used was Russian GOST 6058-73 spherical powder with a particle size of $20-50 \mu \mathrm{m}$. The amount of smaller size particles $(15-20 \mu \mathrm{m})$ was few times greater than that of the $50 \mu \mathrm{m}$ particles. For the measurement, we used a discharge chamber made of Kaprolon (inside diameter is $20 \mathrm{~mm}$ ) with two brass electrodes of $10 \mathrm{~mm}$ diameter. The electrode ends were spherically shaped (segment height is about $1.5 \mathrm{~mm}$ ) and polished. The electrode spacing was initially $2.5 \mathrm{~mm}$. The electrical measurement system was designed as follows. We used a "LATR" transformer, capable of delivering the voltage in the range of a few $\mathrm{V}$ up to $240 \mathrm{~V}$ AC to the load, and under careful manipulation we could perform a voltage steps smaller than one volt. Then we connected its output to the first winding of a

\footnotetext{
* corresponding author; e-mail: kolosenok@gmail.com
}

neon sign transformer (its voltage step-up ratio is about 45 times) in series with a $15 \Omega$ resistive load, in order to limit the current in the case of saturation of the neon sign transformer core after breakdown occurs. When we tried the powder at the specified electrode separation distance, immediate breakdown occurs. Therefore we separated the electrodes to a distance of about 5.8-6 $\mathrm{mm}$ (the exact distance varied for different tests). This creates a non-uniform $E$-field. See the photo of spark gap in Fig. 1. The breakdown was monitored by current measurement in the circuit of the low-voltage winding of the neon sign transformer. When breakdown occurs, the current of a few hundreds of $\mathrm{mA}$ develops. The voltage across the spark gap was monitored with a voltage divider, which has the voltage division ratio of 72.5 times.

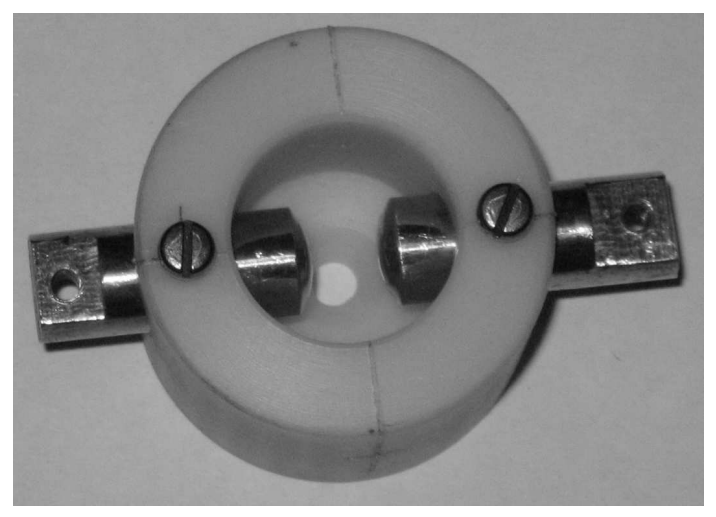

Fig. 1. The spark gap we have used for the tests. Electrode diameter is $10 \mathrm{~mm}$, gap size is $6 \mathrm{~mm}$. 


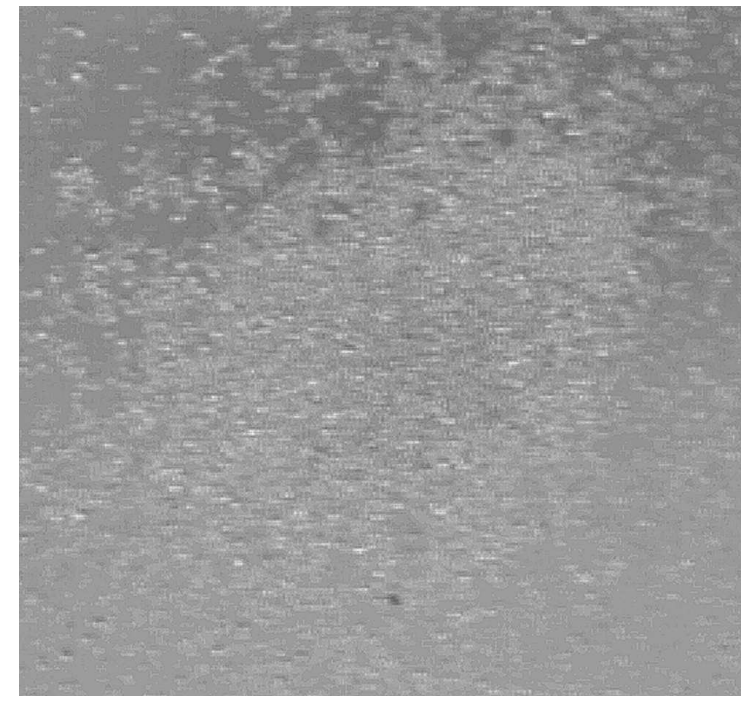

Fig. 2. Spherical $\mathrm{Al}$ powder, particle size $20-50 \mu \mathrm{m}$.

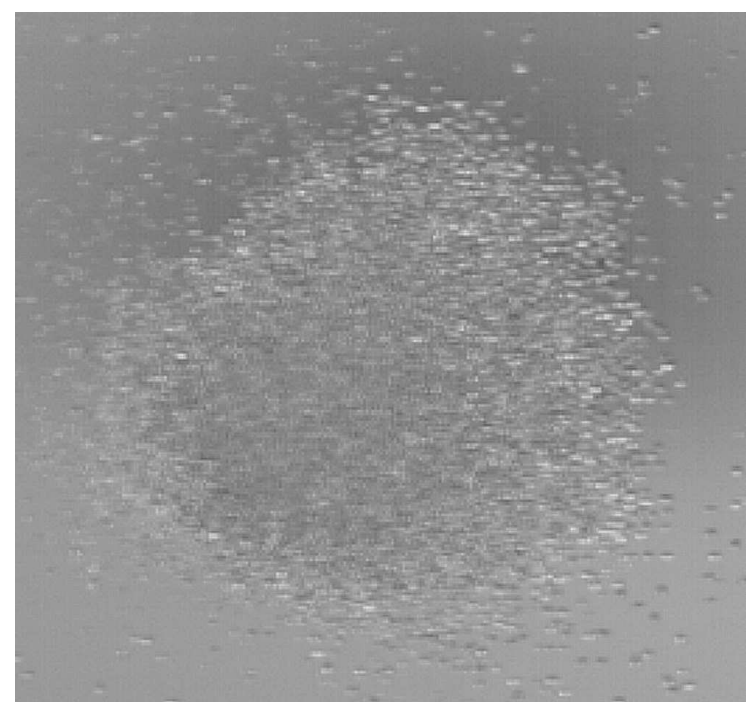

Fig. 3. The Al powder, chemically processed.

In the tests, we used also an $\mathrm{Al}$ powder coated with a Shellac binder. It was prepared by addition of an alcoholic solution of Shellac to the powder and drying it with periodically mixing to avoid complete solidification. After the drying the pieces of composite were gently ground. We prepared two types of Shellac/Al composites - approximately $0.1 \% \mathrm{wt}$ and $1 \%$ wt. They had grain sizes ranging from 100 to $500 \mu \mathrm{m}$ approximately. The shape of the grains was irregular. Also, we prepared a solidified $5 \%$ composite, but the tests in which it was used were unsuccessful (no greater breakdown strength, than that of a pure $\mathrm{Al}$ powder). We call these powders Sh0.1\%, Sh1\% and Sh5\% (solid). Then 5\% solid composite was ground down to, at least, fraction of a $1000 \mu \mathrm{m}$ or smaller, and the better results were obtained, which are similar to those of $\mathrm{Sh} 1 \%$.
Also, we have relatively successful results using a mix of $1 \%$ Shellac grains with $\mathrm{CuO}$ analytical grade powder $50 \%$ to $50 \%$ vol. We call it "Al $+\mathrm{CuO}$ ". The after-breakdown voltage did not decrease to zero, but it was just 3-4 times lower than the pre-breakdown voltage, unlike all the other tests. Therefore, the mechanism of breakdown may be different, probably combined with surface discharge, which we did not investigate.

The attempts to add a few per cents in vol. of Transformer oil to Sh1\% powder suddenly decreased its results to that of an ordinary non-processed powder. That may be due to water contents in the oil or due to Shellac solubility in it, which may destroy the protective layer.

We were also aware about the limitations of the oxide layer that naturally coats the $\mathrm{Al}$ powder and have tried to obtain powders with artificially strengthened dielectric coating. We used a variety of chemicals to do this. Finally, one combination suddenly worked as needed and we processed a few grams of powder. The powder was then washed on filter paper with a distilled water and dried. The resulting powder has a slightly darker color than the initial powder, and appears to be of the same shape and size (see Figs. 2, 3). We shall call it " $\mathrm{Al}+\mathrm{Al}_{2} \mathrm{O}_{3}$ ", while the actual chemical content of the protective layer is unknown and can contain water insoluble salts, other than Al, metal oxides, etc. The more detailed research is still necessary before we can draw certain conclusions about the technological availability of such a product.

\section{The test results}

The following tables summarize the test results. At least 5 measurements were taken for each powder. First row of the table is the test number, the second is the voltage in volts, measured across the output of the voltage divider exactly before the breakdown.

TABLE I

Initially taken $\mathrm{Al}$ powder.

\begin{tabular}{c|c|c|c|c}
\hline \hline 1 & 2 & 3 & 4 & 5 \\
\hline 12.8 & 9.8 & 11.5 & 7.9 & 10
\end{tabular}

TABLE II

The composite "Sh0.1\%".

\begin{tabular}{c|c|c|c|c|c|c|c}
\hline \hline 1 & 2 & 3 & 4 & 5 & 6 & 7 & 8 \\
\hline 15.8 & 10 & 11.4 & 8 & 8.4 & 10.2 & 13 & 13.2 \\
\hline \hline 9 & 10 & 11 & 12 & 13 & 14 & 15 & 16 \\
\hline 15.5 & 15.2 & 14.4 & 11.4 & 13 & 18 & 12.2 & 17
\end{tabular}

For the $\mathrm{Al}+\mathrm{CuO}$ powders mix the breakdown voltage values were in the range of $27-36 \mathrm{~V}$. The pure $\mathrm{CuO}$ had the following breakdown strength value: $39 \mathrm{~V}$. 
TABLE III

The composite

"Sh1\%".

\begin{tabular}{c|c|c|c|c}
\hline \hline 1 & 2 & 3 & 4 & 5 \\
\hline 13 & 18 & 27 & 18.5 & 13
\end{tabular}

TABLE IV

The chemically produced powder "Al $+\mathrm{Al}_{2} \mathrm{O}_{3}$ ".

\begin{tabular}{c|c|c|c|c}
\hline \hline 1 & 2 & 3 & 4 & 5 \\
\hline 53 & 66 & 73 & 79 & 75
\end{tabular}

\section{Conclusions}

As we can see from the data, the leading performance material is chemically processed powder. Its average breakdown strength is as high as $5 \mathrm{kV} / \mathrm{cm}$, which is 6.6 times greater than that of source powder. Also, when fired sequentially 5 times, the powder exhibited a growth in its electrical breakdown strength, unlike the source powder. This special ability can probably be developed for the usage in quenching switches. Anyway, if extensive tests will be done, we may obtain new materials for the shock wave electrophysical devices, such as flux compression generators and switches [2].

\section{References}

[1] S.V. Kolosenok, D. Hemmert, S. Holt, in: Proc 12th Int. Conf. on Megagauss Magnetic Field Generation and Related Topics, Novosibirsk (Russia) 2008.

[2] D. Hemmert, S. Holt, J. Krille, in: Proc. 10th Annual Directed Energy Symposium, Huntsville (AL) 200\%, Directed Enerergy Professional Society, Albuquerque (NM) 2007, p. 1632. 